EMBRYARIDDLE
Aeronautical University

SCHOLARLY COMMONS
International Journal of Aviation, Aeronautics, and Aerospace

\title{
Computation of Eclipse Time for Low-Earth Orbiting Small Satellites
}

Sumanth R M

R.V. College of Engineering, sumanth.rm000@gmail.com

Follow this and additional works at: https://commons.erau.edu/ijaaa

Part of the Astrodynamics Commons

\section{Scholarly Commons Citation}

R M, S. (2019). Computation of Eclipse Time for Low-Earth Orbiting Small Satellites. International Journal of Aviation, Aeronautics, and Aerospace, 6(5). https://doi.org/10.15394/ijaaa.2019.1412

This Article is brought to you for free and open access by the Journals at Scholarly Commons. It has been accepted for inclusion in International Journal of Aviation, Aeronautics, and Aerospace by an authorized administrator of Scholarly Commons. For more information, please contact commons@erau.edu. 


\section{Computation of Eclipse Time for Low-Earth Orbiting Small Satellites}

\section{Cover Page Footnote}

This paper is a bonafide work of engineers of RVSAT-1 


\section{Introduction}

With the emergence of small-sized satellites (under $500 \mathrm{~kg}$ ), space has become accessible to a greater number of technical institutions due to their reduced cost and complexity. In this context, R.V. College of Engineering, Bengaluru has initiated a student nanosatellite project called RVSAT-1. RVSAT1 which is a $2 \mathrm{U}$ nanosatellite aims to conduct a micro-biological experiment to analyze the growth of a micro-organism in space environment. For a small satellite, the demand for more power with lower mass and volume is continuously increasing. A satellite orbiting earth passes through a shadow region where the solar arrays are deprived of solar illumination. Depending upon the type of orbit, the time duration in shadow region varies. This time duration is called eclipse time and the shadow region is called eclipse region. In eclipse region, the satellite uses secondary power source (usually batteries) to power the various subsystems in the satellite. Thus, computation of eclipse time becomes very important for sizing of batteries and to maintain constant heat balance within the satellite. MATLAB framework was used to perform the orbital calculations and to obtain more apt and reliable results.

\section{Assumptions}

The computation of eclipse time was carried out assuming that the planet is spherical in shape. A planet's shadow consists of two distinct conical projections: The Umbra and the Penumbra as shown in Figure 1. However, the umbral region has been treated as cylindrical projection of Earth (for simpler calculations). The assumptions are fairly accurate for low altitude circular orbits but may lead to significant errors for high altitude or highly elliptical orbits.

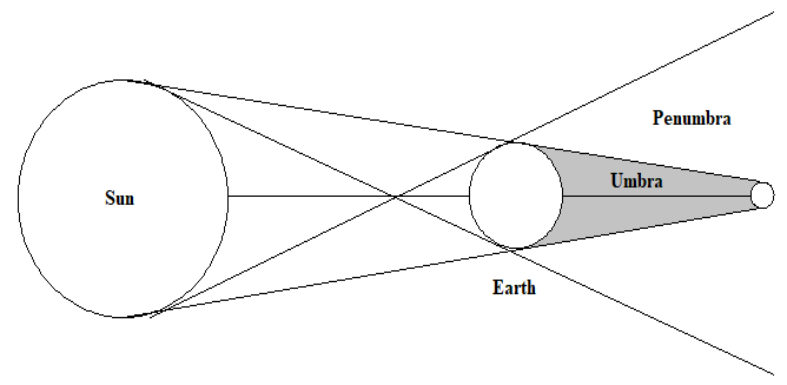

Figure 1. Earth's shadow showing umbra and penumbra region Shadow Analysis Methodology.

The eclipse duration is mainly the function of altitude, size of earth and the orbital beta angle. Whilst assuming spherical shape of the planet, the effects of non-spherical shape are accounted for only in the sense that they perturb the orbit. The following method uses JPL DE405 Ephemeris data to compute the eclipse duration. The seasonal variation due to solar motion and perturbations of the orbit, 
both leads to the variation of $\beta$ angle, thus considering both the above parameters is essential.

\section{The Beta-Angle Calculation}

The orbital beta angle $(\beta)$ is defined as the angle between the solar vector and its projection onto the orbital plane. To calculate the beta angle, it is necessary to determine the angle between the unit solar vector $(\hat{s})$ and the unit vector normal to the orbital plane $(\hat{n})$. Figure 2 shows the beta angle $(\beta)$ and the angle between solar vector and normal vector, which is given by:

$\Phi=\frac{\pi}{2}+\beta$

Figure 2. Orbital beta angle.

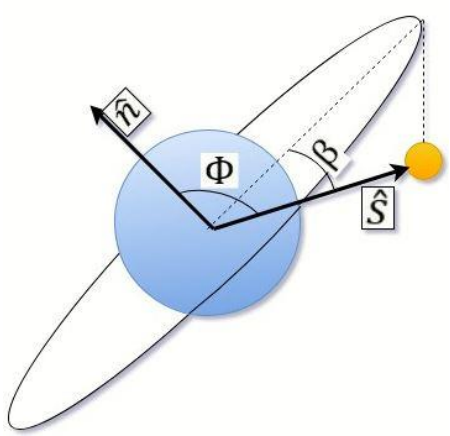

It is possible to get the unit solar vector $(\hat{\boldsymbol{s}})$ and unit normal vector $(\widehat{\boldsymbol{n}})$ using two Euler angle transformations each, the sequence of the transformations is described below:

In the celestial inertial co-ordinate system, solar vector $(\hat{S})$ points towards the sun is governed by two parameters: Right Ascension of Sun $(\Gamma)$ and Declination of Sun $(\varepsilon)$. Similarly, we define a vector $\hat{n}$, as the vector pointing normal to the orbital plane and is governed by two parameters: Orbital inclination (i) and Right Ascension of Ascending Node $(\Omega)$.

For the solar vector $(\vec{s})$, from the Figure 3,

1. A rotation of the unit vector of declination of the sun $(\varepsilon)$ about the $\mathrm{x}$-axis.

2. A rotation of right ascension of the sun $(\boldsymbol{\Gamma})$ about the new z-axis. 


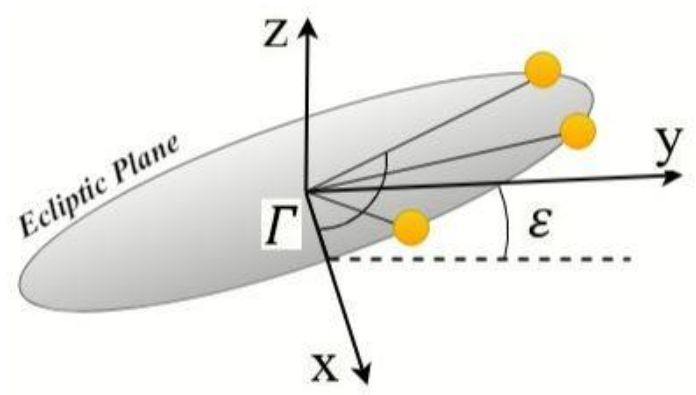

Figure 3. Euler angle transformation for solar vector.

Mathematically,

$\hat{s}=\left[\begin{array}{ccc}1 & 0 & 0 \\ 0 & \cos \varepsilon & -\sin \varepsilon \\ 0 & \sin \varepsilon & \cos \varepsilon\end{array}\right]\left[\begin{array}{ccc}\cos \Gamma & -\sin \Gamma & 0 \\ \sin \Gamma & \cos \Gamma & 0 \\ 0 & 0 & 1\end{array}\right]\left\{\begin{array}{l}1 \\ 0 \\ 0\end{array}\right\}$

$\hat{s}=\left[\begin{array}{c}\cos \Gamma \\ \cos \varepsilon \cdot \sin \Gamma \\ \sin \varepsilon \cdot \sin \Gamma\end{array}\right]$

Similarly, for normal vector ( $\hat{\mathrm{n}})$, from the Figure 4,

1. A rotation of the unit vector of the Right ascension of ascending node $(\Omega)$ about the z-axis.

2. A rotation of inclination $(i)$ about the new $\mathrm{z}$-axis.

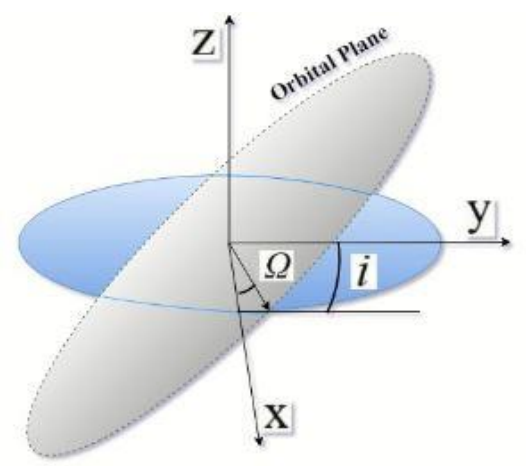

Figure 4. Euler angle transformation for normal vector. 
Mathematically,

$\hat{n}=\left[\begin{array}{ccc}\cos \Omega & -\sin \Omega & 0 \\ \sin \Omega & \cos \Omega & 0 \\ 0 & 0 & 1\end{array}\right]\left[\begin{array}{ccc}1 & 0 & 0 \\ 0 & \operatorname{cosi} & -\sin i \\ 0 & \sin i & \operatorname{cosi}\end{array}\right]\left\{\begin{array}{l}0 \\ 0 \\ 1\end{array}\right\}$

$\hat{n}=\left[\begin{array}{c}\operatorname{sini} \cdot \sin \Omega \\ -\operatorname{sini} \cdot \cos \Omega \\ \cos i\end{array}\right]$

We also observe that, $\cos \phi=(\hat{s}) \cdot(\hat{n})$

The expression for beta angle $(\beta)$ is given by:

$\beta=\sin ^{-1}(\cos \Gamma \sin \Omega \sin i-\sin \Gamma \cos \varepsilon \cos \Omega \sin i+\sin \Gamma \sin \varepsilon \cos i)$

\section{Variation of Beta Angle}

For any satellite, $\beta$ will vary continuously with time because of the orbital perturbation and seasonal variation of solar declination $(\varepsilon)$ and Right Ascension of Sun $(\Gamma)$. The orbital perturbation due to the planet's oblateness is accounted for, by calculating the rate of change of RAAN $(\dot{\Omega})$ which is expressed as follows:

$\dot{\Omega}=-\frac{3}{2} J_{2} v\left(\frac{R_{e}}{\left(R_{e}+h\right)\left(1-\mathrm{e}^{2}\right)}\right) \cos i$

Where $\dot{\Omega}$ is expressed in degrees/day and $v$ is the Mean motion which is expressed as:

$v=\sqrt{\frac{G M}{\left(R_{e}+h\right)^{3}}}$

As viewed from the sun, an orbit with $\beta$ equal to $0^{0}$ has the longest eclipse time because of shadowing by the full diameter of Earth. As $\beta$ increases the satellite is sun facing for a larger percentage of each orbit, thus decreasing in eclipse duration. With $\beta$ equal to $90^{\circ}$, no eclipses exist at any altitude. The absolute value of beta varies as $|\beta| \leq(i+23.45)$.

\section{Eclipse-Time Calculation}

The eclipse duration is determined by multiplying eclipse fraction $\left(f_{e}\right)$ with total time period. The eclipse fraction $\left(f_{e}\right)$ is calculated as follows: 
$f_{e}= \begin{cases}\frac{1}{180^{0}} \cos ^{-1}\left[\frac{\sqrt{h^{2}+2 \operatorname{Re} h}}{(\operatorname{Re}+h) \cos \beta}\right] & \text { if }|\beta|<\beta^{*} \\ 0 & \text { if }|\beta| \geq \beta^{*}\end{cases}$

Total Time-Period $(T)=2 \pi \sqrt{\frac{(\mathrm{Re}+h)^{3}}{G M}}$

Eclipse Time $(T \mathrm{e})=T \cdot f_{e}$

$\beta^{*}=\sin ^{-1}\left(\frac{\mathrm{R}_{\mathrm{e}}}{\mathrm{Re}+\mathrm{h}}\right)$

Where, $\beta^{*}$ is the angle at which the eclipse begins.

\section{Discussion and Results}

In this section simulation results have been presented which was computed using MATLAB framework. The results include variation of eclipse duration and $\beta$ angle as a function of altitude and inclination.

From the Figure 5 it is evident that for LEO as the altitude increases the eclipse fraction decreases. The decrease in eclipse time allows for higher power generation by solar array itself and this reduces the reliance on batteries as secondary power source. In addition, Figures $6-10$ depict the variation of eclipse time (in minutes) at inclination of $0^{\circ}, 30^{\circ}, 75^{\circ}, 90^{\circ}$ and $120^{\circ}$ respectively. For LEO the maximum eclipse duration remains close to 35 minutes. Moreover, for lower inclination orbits the average eclipse time over the year is very high but as the inclination increases the average eclipse duration over the year decreases. Thus, as shown in figure 11 orbits with $90^{\circ}<i<120^{\circ}$ have minimum average eclipse duration over the year than compared to orbits with lower inclination. Also, as shown in figure 12-14 with the increase in inclination the range of $\beta$ angle increases. So, increasing beta angle results in increased solar illumination thus decreasing the eclipse duration. However, for a particular inclination the range of $\beta$ angle remains constant at any altitude. The change in beta angle directly affects the thermal conditions of the satellite. The increase in $\beta$ angle increases the heat loads on the satellite. Based on variation of heat loads the thermal components are used to maintain the optimum heat balance within the satellite.

\section{Conclusions}

In this paper, a method to compute the eclipse time using MATLAB framework has been developed. The $\beta$ angle and eclipse fraction were computed through Euler transformations and mathematical equations respectively. From the 
obtained results it was evident that orbits with $90^{\circ}<\mathrm{i}<120^{\circ}$ have the minimum average eclipse duration. Having lesser eclipse duration gives an advantage of having a battery with lesser specific energy thus reducing mass and cost. Also, by knowing the eclipse time, the satellite can be designed to withstand the varying thermal loads, thus maintaining optimum thermal conditions required for the satellite. From the calculations it was evident that for Low-Earth circular orbits, penumbral duration is very less compared to the umbral duration. However, for high altitude or highly elliptical orbits the penumbral region can't be ignored. Also, further research can be done to compute eclipse time for elliptical orbits. In future, we intend to design and develop complete payload configuration for RVSAT-1. 


\section{References}

Cunningham, F.G. (1962). Calculation of the eclipse factor for elliptical satellite orbits. Retrieved from https://ntrs.nasa.gov/search.jsp?R=19630000622

Gilmore, D. G., \& Donabedian, M. (Eds.). (2003). Spacecraft thermal control handbook: Cryogenics (vol. 2). E1 Segundo, CA: The Aerospace Press.

Ismail, M. N., Bakry, A., Selim, H. H., \& Shehata, M. H. (2015). Eclipse intervals for satellites in circular orbit under the effects of Earth's oblateness and solar radiation pressure. NRIAG Journal of Astronomy and Geophysics, 4(1), 117-122.

Larson, W. J., \& Wertz, J. R. (1992). Space mission analysis and design (No. DOE/NE/32145-T1). Torrance, CA: Microcosm, Inc.

Longo, C.R.O., \& Rickman, S. L. (1995). Method for the calculation of spacecraft umbra and penumbra shadow terminator points. Washington, DC: National Aeronautics and Space Administration.

Mocanu, B., Burlacu, M. M., Kohlenberg, J., Prathaban, M., Lorenz, P. \& Tapu, R. (2009, July). Determining optimal orbital path of a nanosatellite for efficient exploitation of the solar energy captured. In 2009 First International Conference on Advances in Satellite and Space Communications (pp. 128-133). IEEE.

Patel, M. R. (2004). Spacecraft power systems. Boca Raton, FL: CRC press.

Rickman, S. L. (2014, August). Introduction to on-orbit thermal environments. In Thermal and Fluids Analysis Workshop. Retrieved from https://www.nasa.gov/nesc/technicalpapers

Vallado, D. A. (2010). Orbital mechanics fundamentals. ISBN-13: 9781881883142 


\section{Appendix}

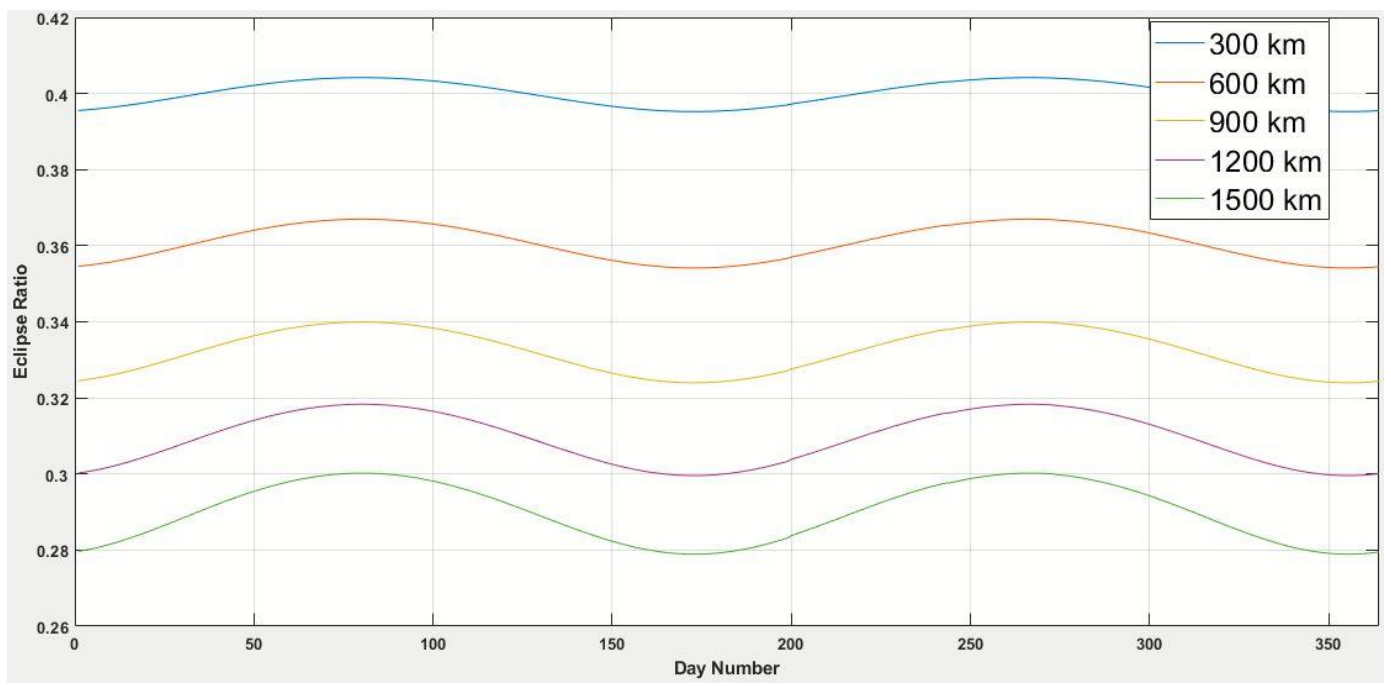

Figure 5. Variation of eclipse fraction as a function of altitude.

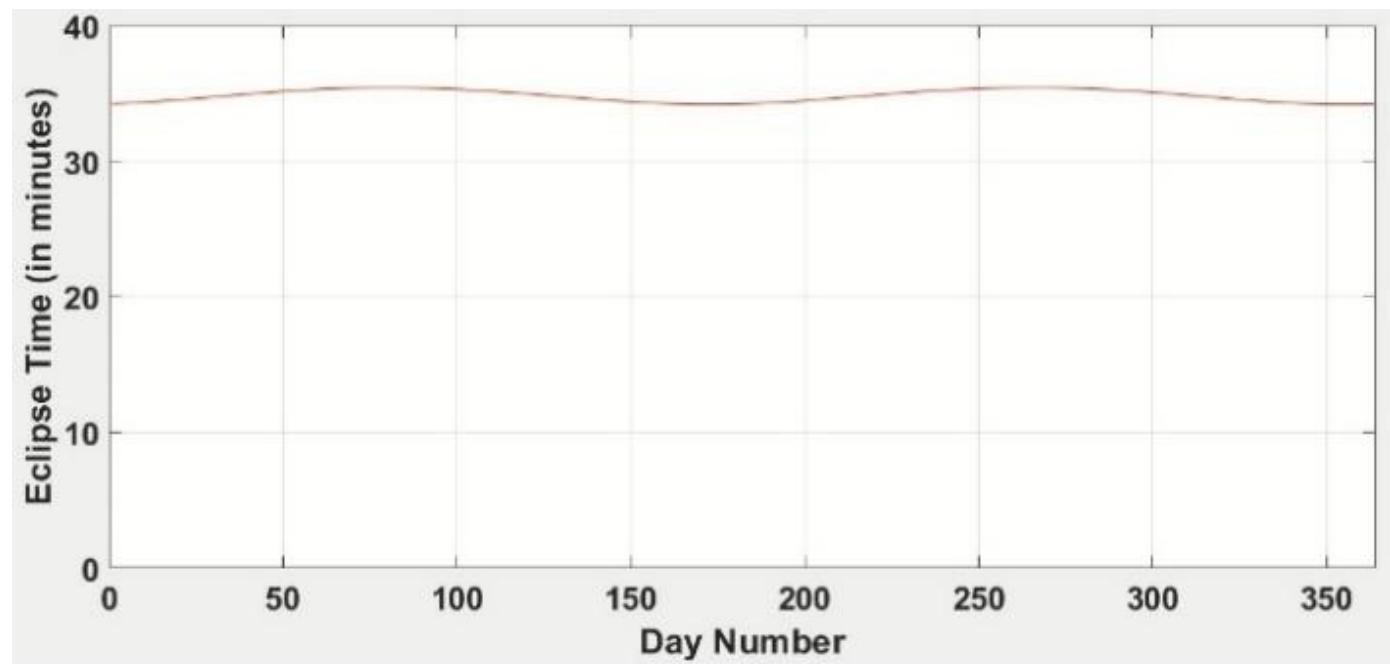

Figure 6. Variation of eclipse time at $i=0^{0}$. 
R M : Computation of Eclipse Time for LEO Small Satellites

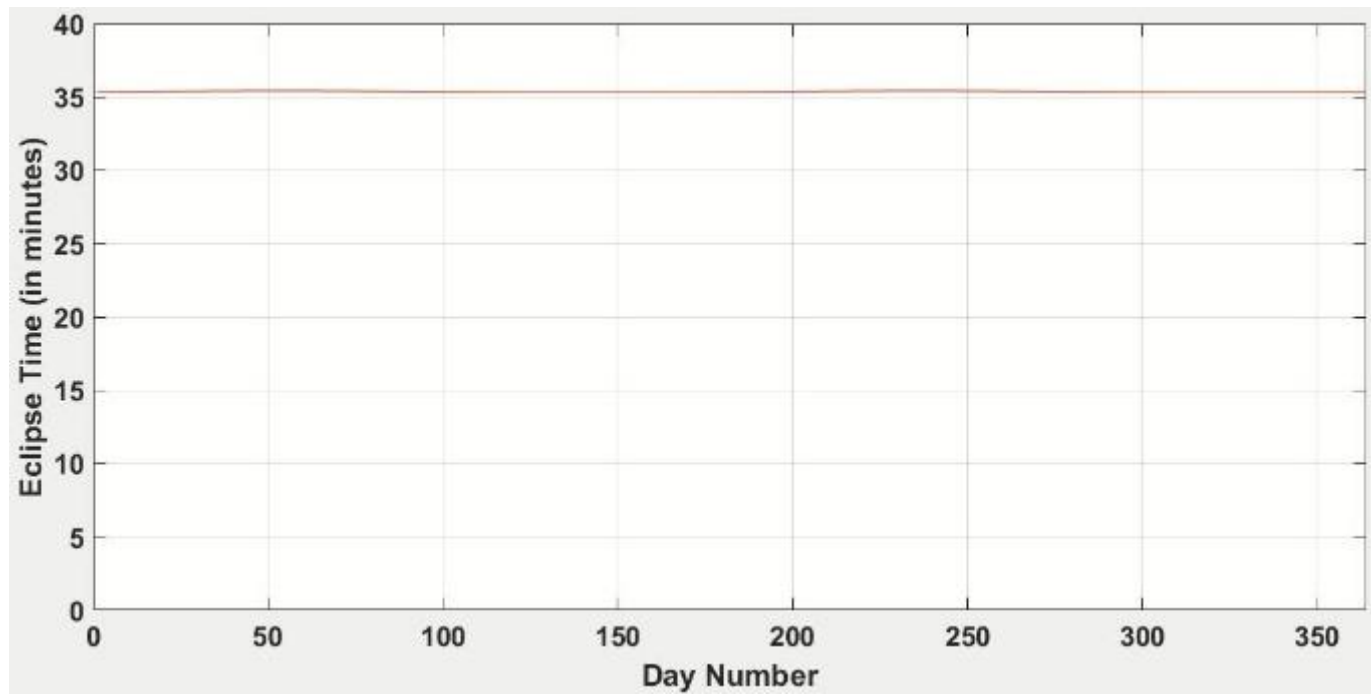

Figure 7. Variation of eclipse time at $i=30^{\circ}$.

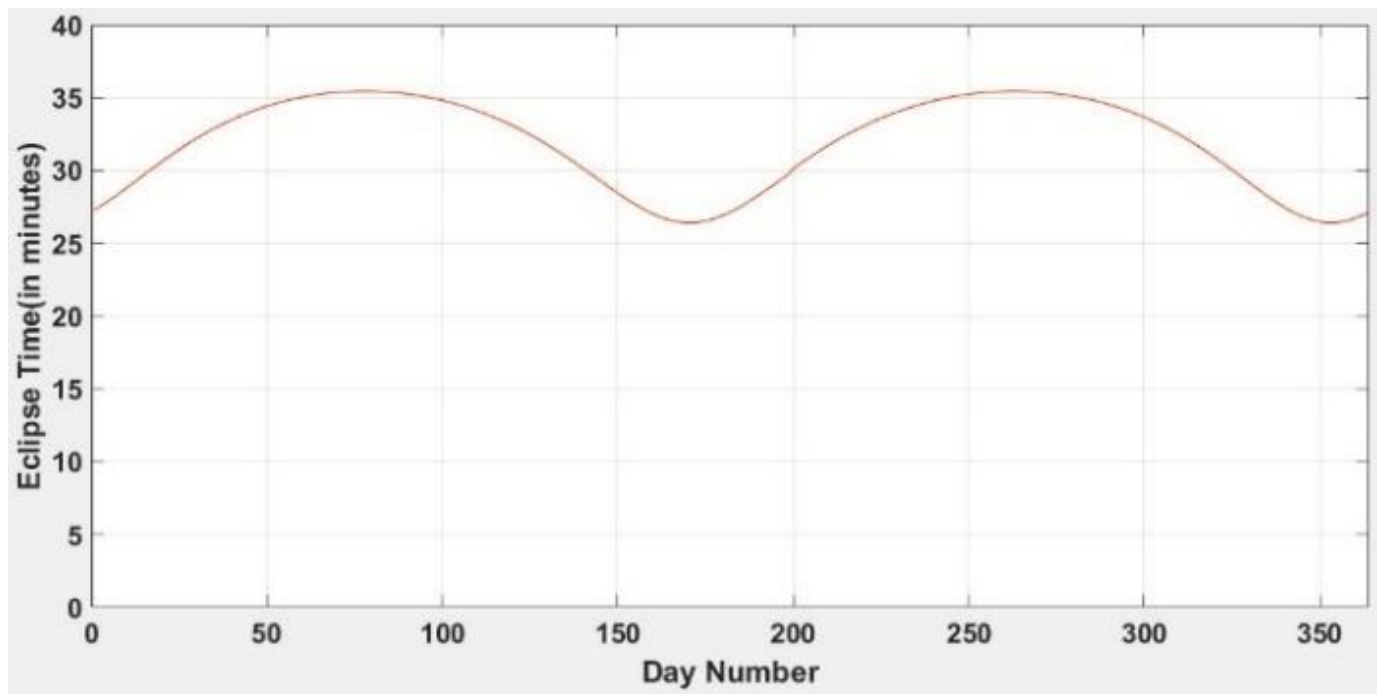

Figure 8. Variation of eclipse Time at $i=75^{0}$. 
International Journal of Aviation, Aeronautics, and Aerospace, Vol. 6 [2019], Iss. 5, Art. 15

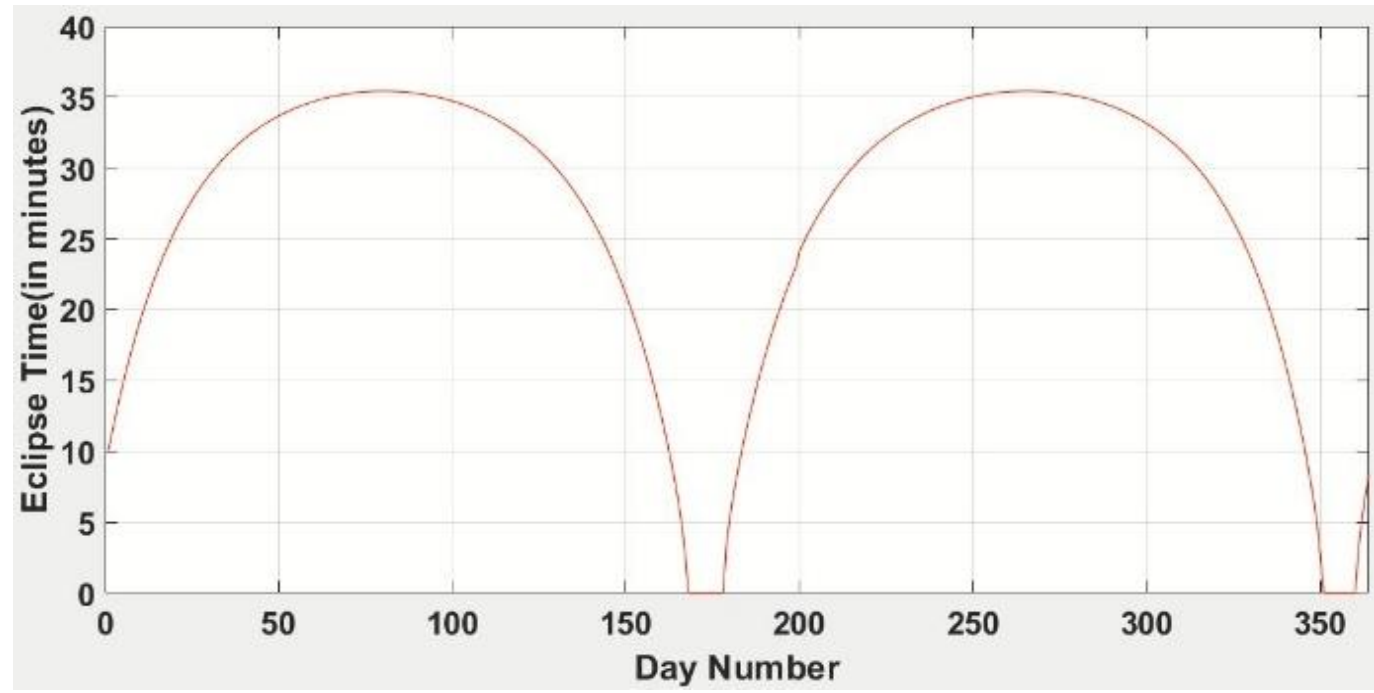

Figure 9. Variation of Eclipse Time at $i=90^{\circ}$.

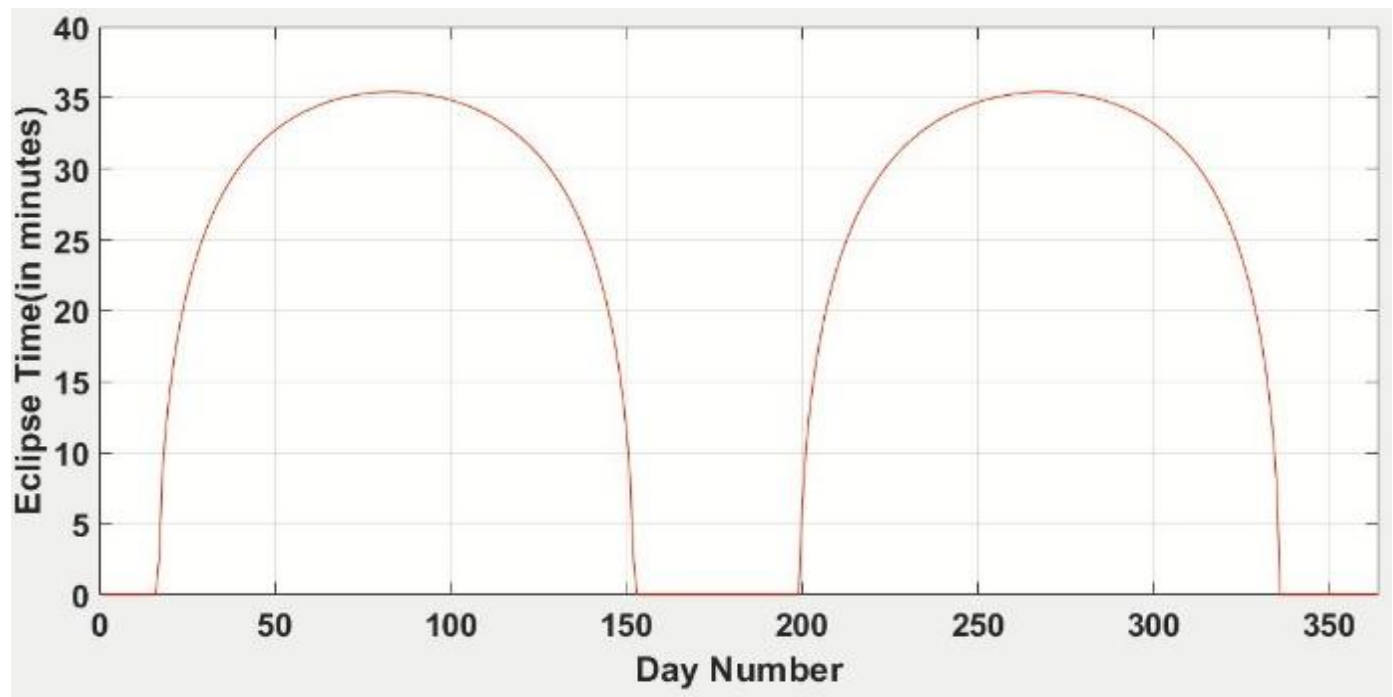

Figure 10. Variation of eclipse time at $i=120^{\circ}$. 
R M : Computation of Eclipse Time for LEO Small Satellites

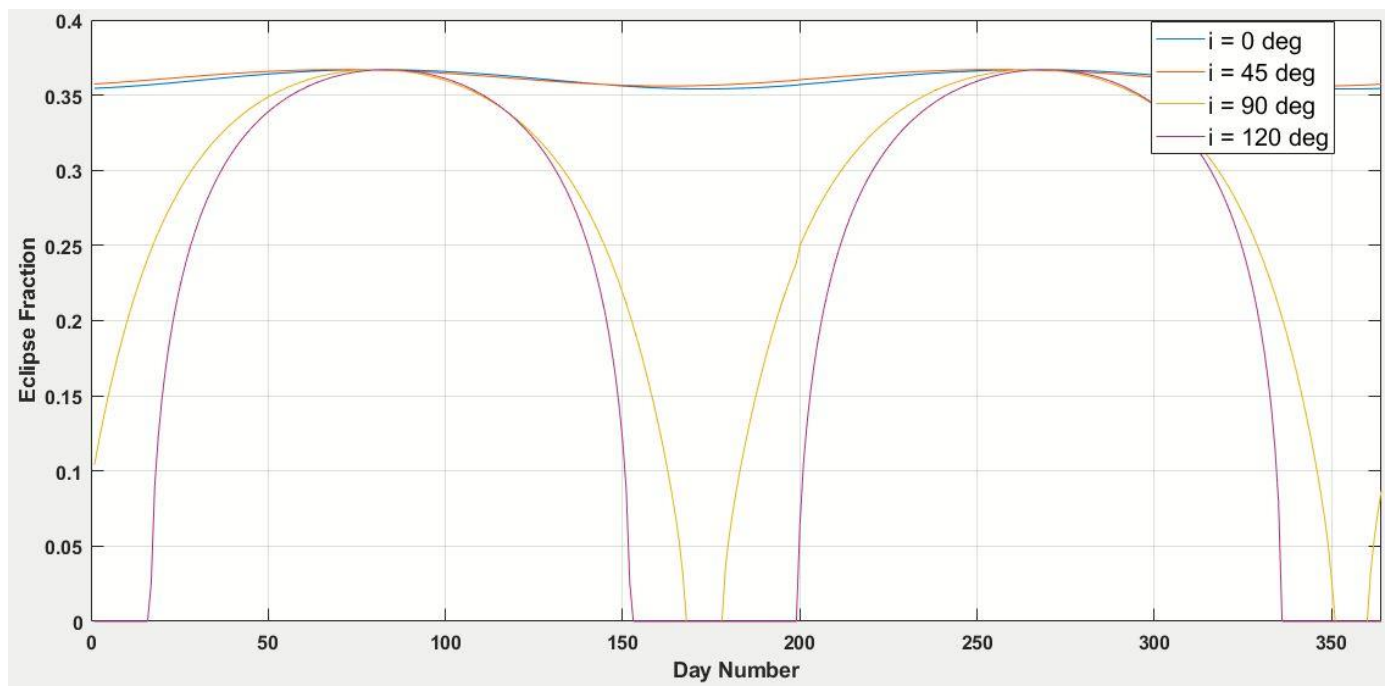

Figure 11. Variation of eclipse fraction at different inclination.

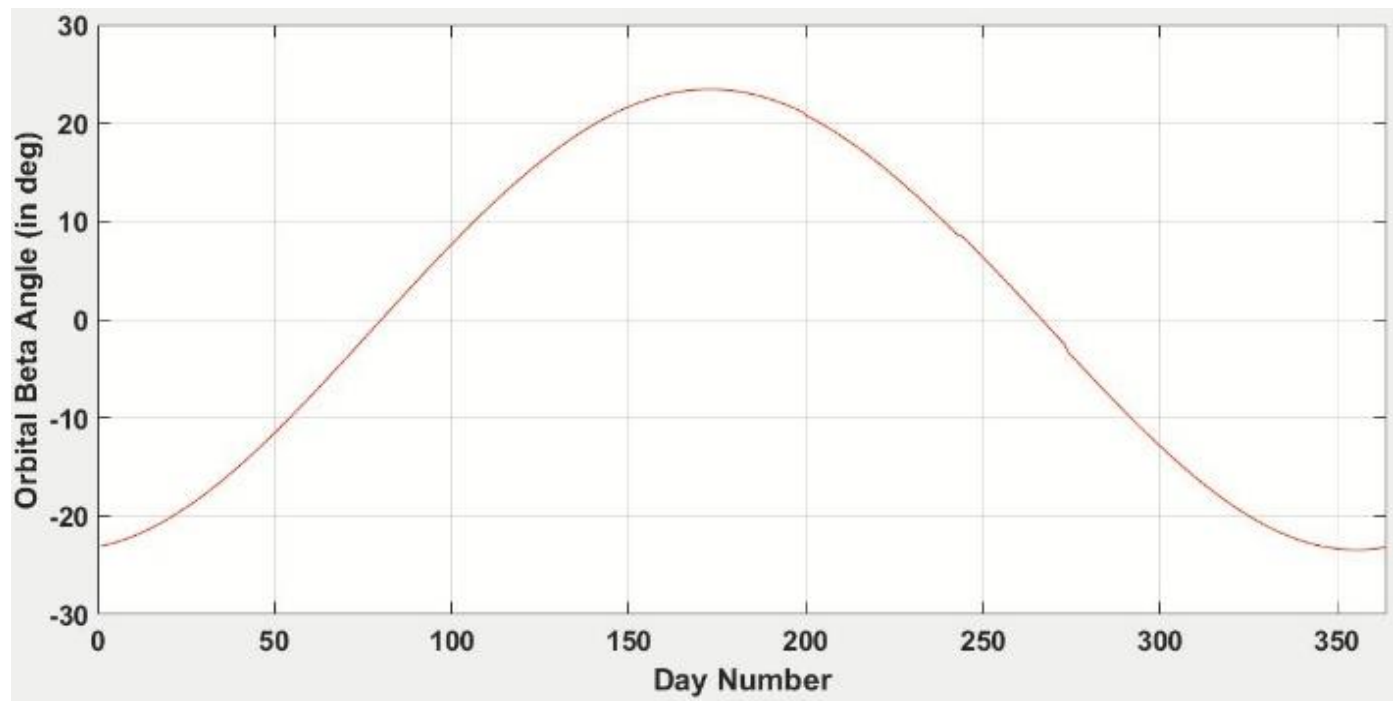

Figure 12. Variation of beta angle at $i=0^{0}$. 


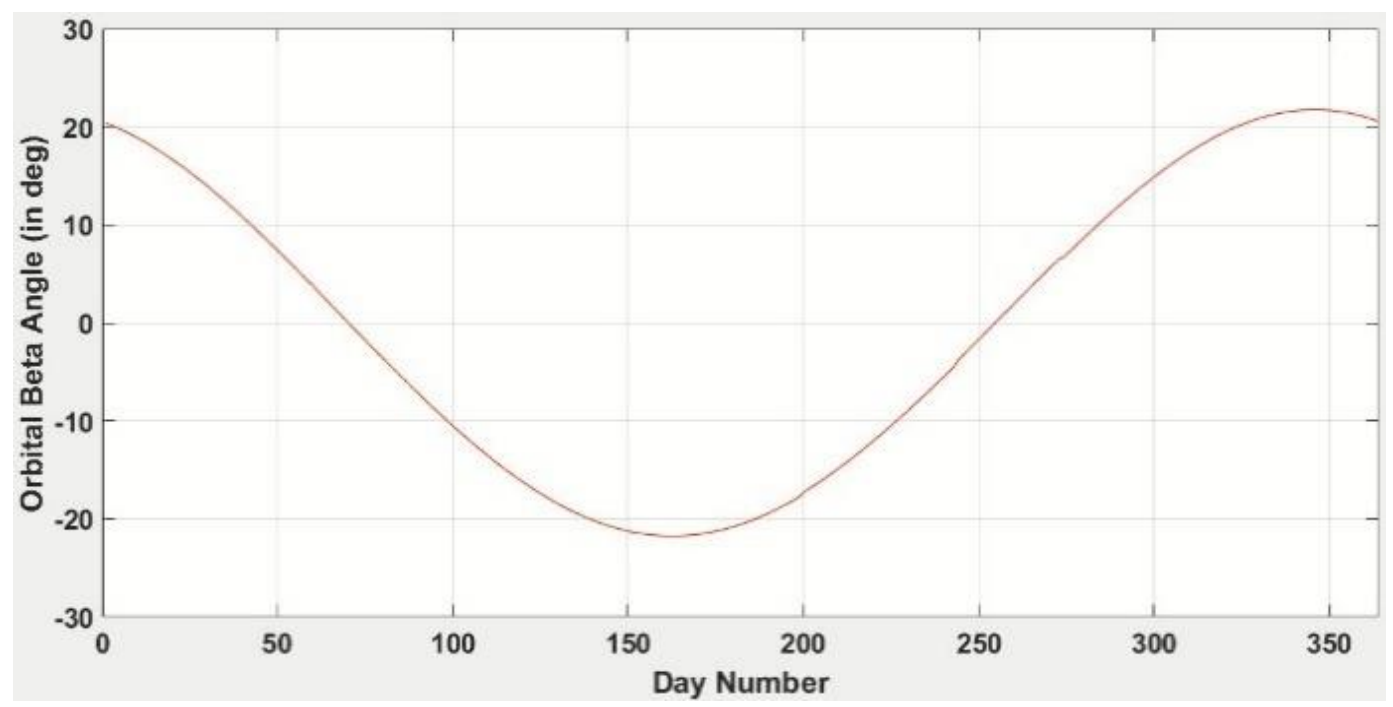

Figure 13. Variation of beta angle at $i=45^{\circ}$.

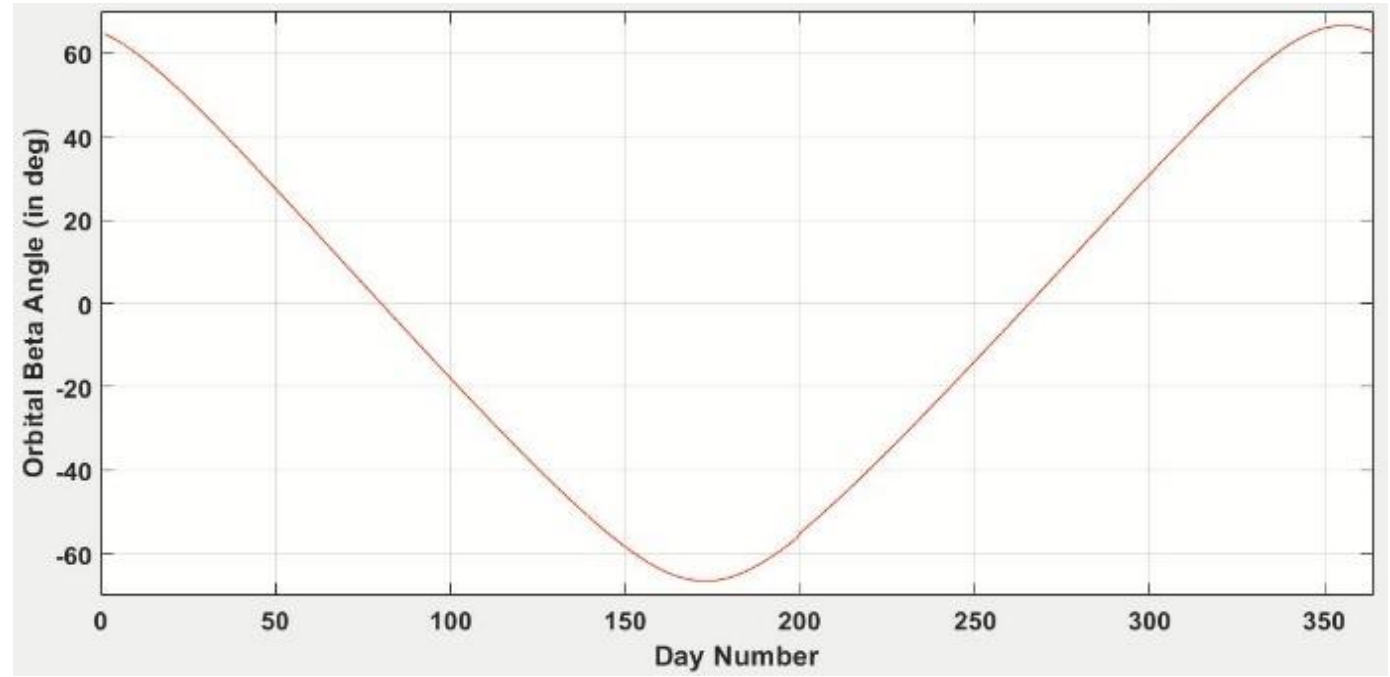

Figure 14. Variation of beta angle at $i=90^{\circ}$. 


\section{General Terms}

$\beta$ - Orbital Beta angle

$\hat{s}$ - Unit solar vector

$\hat{n}$ - Unit normal vector

$\Phi$ - Angle between the unit solar vector $(\hat{s})$ and the unit normal vector $(\hat{n})$.

$\Gamma$ - Right Ascension of Sun

$\varepsilon$ - Declination of Sun

$i$ - Orbital inclination

$\Omega$ - Right Ascension of Ascending Node

e - Eccentricity

$J_{2}$ - Oblateness perturbation coefficient of earth $=0.00108263$.

$f_{e}$ - Eclipse fraction

$T$ - Orbital Time Period

Te - Eclipse Time

$G$ - Universal Gravitational Constant

$M$ - Mass of Earth

$h$ - Altitude of satellite

$R_{e^{-}}$Equatorial Radius of Earth

$v$ - Mean Motion of satellite 